Ebisu Ebisu

Études japonaises Études japonaises

47 | printemps-été 2012

Catastrophes du 11 mars 2011, désastre de

Fukushima : fractures et émergences

\title{
Milieu, co-suscitation, désastres naturels et
} humains

The Co-Arousing Relationship Between Natural and Human Disasters in Human Milieux

風土と縁起亡天災・人桨

\section{Augustin Berque}

\section{(2) OpenEdition}

Journals

Édition électronique

URL : http://journals.openedition.org/ebisu/234

DOI : 10.4000/ebisu.234

ISSN : 2189-1893

Éditeur :

Institut français de recherche sur le Japon (UMIFRE 19 MAEE-CNRS), Maison franco-japonaise

Édition imprimée

Date de publication : 1 juin 2012

Pagination : 41-48

ISSN : $1340-3656$

\section{Référence électronique}

Augustin Berque, «Milieu, co-suscitation, désastres naturels et humains », Ebisu [En ligne], 47 | printemps-été 2012, mis en ligne le 03 avril 2014, consulté le 22 avril 2019. URL : http:// journals.openedition.org/ebisu/234; DOI : 10.4000/ebisu.234 


\title{
Milieu, co-suscitation, désastres naturels et humains
}

\author{
Augustin BERQUE
}

\section{Phénomènes et catastrophes}

"Co-suscitation » traduit ici engi, prononciation japonaise des sinogrammes 縁起 (yuánqŭ) par lesquels fut rendu le sanscrit pratītya samutpāda. Ce concept est plus habituellement traduit, à partir du sanscrit, par « coproduction conditionnée "; mais il est possible, à partir des sinogrammes, de le rendre par co (縁) susciter (起). L'idée, c'est que les phénomènes se conditionnent les uns les autres, et qu'en ce sens, ils se co-suscitent.

Il s'agit là d'abord d'un concept bouddhique, avec en arrière-plan une logique et une cosmologie très élaborées. En japonais toutefois, l'usage ordinaire de ce terme relève de la superstition. Par exemple, engi ga warui 縁起が悪い, “c'est mauvais signe »; engi o katsugu 縁起を担ぐ, " porter de l'engi ", c'est être superstitieux. Dans ce sens-là, il s'agirait d'identifier les mauvais présages qui auraient laissé prévoir la catastrophe du San-ichi-ichi 三一, le «Trois-un-un », i.e. la catastrophe du 11 mars (2011); mais c'est dans le sens originel que l'on entendra ici la co-suscitation : à savoir comment facteurs humains et facteurs naturels se sont réciproquement conditionnés dans le San-ichi-ichi.

Géographe, orientaliste, philosophe, Augustin Berque est membre de l'Académie européenne. Il a été en 2009 le premier occidental à recevoir le Grand Prix de Fukuoka pour les cultures d'Asie. 
La manière ordinaire de concevoir cet événement relève de la causalité matérielle. Le séisme serait la cause première, et le reste en serait l'effet. Les séismes étant un fait géophysique, lequel ne doit rien aux affaires humaines, il serait donc impensable, en pareille matière, de parler de co-suscitation.

Le point de vue est ici différent. La géophysique s'occupe de phénomènes naturels, pas de catastrophes. En eux-mêmes, les phénomènes naturels, quelle que soit leur échelle (d'un choc de particules à un choc de galaxies), ne sont pas des catastrophes; ils arrivent, c'est tout. Ici en revanche, il s'agit d'une catastrophe, c'est-à-dire de quelque chose qui implique l'existence humaine. Cela ne signifie pas seulement que le phénomène en question exerce un effet matériel sur celle-ci (quantifiable en nombre de morts, par exemple) ; mais aussi que, du fait de l'existence humaine, ledit phénomène est une catastrophe. Il doit ce qu'il est à l'existence humaine. En d'autres termes, il devient une réalité humaine ; et comme nous ne pouvons être qu'humains, c'est tout simplement la réalité.

Ce n'est pas là une tautologie. Distinguer entre phénomène et catastrophe n'est pas non plus qu'une affaire de mots. En effet, la réalité humaine dont relèvent les catastrophes n'est pas réductible à la réalité physique dont relèvent les phénomènes naturels; elle implique un milieu, pas seulement un environnement.

S’agissant de la réalité humaine, la distinction entre milieu (füdo 風土) et environnement ( $k a n k y o ̄$ 環境) a été établie par Watsuji Tetsurō, avec la publication de Füdo (1935), quasi en même temps que Jakob von Uexküll, avec la publication de Milieux animaux et milieu humain (Streifzüge durch die Umwelten von Tieren und Menschen, 1934), établissait de son côté, pour la réalité animale, la distinction entre Umwelt (le monde ambiant propre à une espèce donnée) et Umgebung (le donné environnemental universel). Ces deux auteurs ont donné par là un souffle nouveau à la mésologie (füdoron 風土論), qui désormais allait pouvoir s'identifier comme l'étude des milieux (vivants en général, humains en particulier), alors que ce terme, initialement proposé par le médecin Charles Robin en 1848, avait un sens qui a été ensuite recouvert par l'écologie, science de l'environnement (Umgebung) et non pas du milieu (Umwelt).

Les catastrophes relèvent du milieu, les phénomènes naturels de l'environnement. Toutes sortes de phénomènes naturels nous échappent, mais pas les catastrophes, parce qu'elles nous concernent. Le séisme du 9 juillet 1958 (M 8,3) en Alaska ne fit pas en lui-même de victimes, mais il entraîna 
dans la baie de Lituya un glissement de terrain qui, comprimant l'eau dans ce fjord, y provoqua une vague de 524 mètres. La région étant quasi déserte, il n'y eut que deux victimes. Du point de vue de l'existence humaine, on ne peut donc pas dire que ce fut une catastrophe, bien que le phénomène fût inouï ; mais pour la forêt locale, ce fut, certes, une catastrophe.

\section{Milieu et co-suscitation}

Que les catastrophes relèvent du milieu entraîne qu' elles relèvent de la cosuscitation ; car sans l'existence humaine, elles ne seraient pas ce qu'elles sont. Voilà qui pourra sembler une lapalissade, mais cela veut dire en fait qu'une telle réalité ne peut pas être saisie dans les termes qui régissent la conception moderne de la réalité ; d'où suit que dans ces termes-là, cette réalité ne peut pas, non plus, être traitée comme il convient. S'agissant de milliers de morts, cela ne peut pas être pris à la légère.

Dans la conception moderne, l'environnement est une réalité objective (une Umgebung). Il y a, entre cet objet et les sujets humains, un abîme ontologique : celui du dualisme. Comme Descartes l'institua paradigmatiquement dans le Discours de la méthode, le cogito individuel, i.e. le sujet moderne, n'a besoin d'aucun lieu pour être. Il existe en lui-même, hors de tout milieu et de toute histoire. Du point de vue mésologique, il en va tout autrement : en réalité, il y a historiquement co-suscitation entre le sujet et le milieu qui lui est propre ; et cela vaut tant à l'échelle individuelle qu'à l'échelle collective.

Celui qui a exprimé le plus clairement ce rapport de co-suscitation n'est ni Uexküll ni Watsuji, mais Imanishi Kinji 今西錦司 dans Seibutsu no sekai 生物の世界 (Le monde du vivant, 1941), où il emploie la formule « subjectivation de l'environnement, environnementalisation du sujet " (kankyō no shutaika, shutai no kankyōka 環境の主体化、主体の環境化). Imanishi ne se référait ni à Uexküll ni à Watsuji, et n'emploie donc pas leurs concepts, mais ce qu'il désigne par là n'est autre que le processus dans lequel l'environnement (kankyō, Umgebung) devient milieu (füdo, Umwelt). Imanishi avait beaucoup lu Nishida Kitarō 西田幾多郎, mais il n'a pas non plus tiré parti de ce que ce dernier, dans Basho 場所 (Le lieu, 1927), a appelé « logique du prédicat" (jutsugo no ronri 述語の論理). Pour Nishida, le monde n'est pas une substance, c'est un prédicat néantiel - un "monde prédicatif " 
(jutsugo sekai 述語世界). Pour simplifier, l’on considérera ici que le monde est l'ensemble des prédicats selon lesquels l'humanité saisit un sujet qui est la nature ; il est donc prédicatif. Sans rapport avec la thèse nishidienne, Maurice Merleau-Ponty, dans Phénoménologie de la perception (1945), devait montrer de son côté que le réel est chargé par notre chair de "prédicats anthropologiques ». Enfin André Leroi-Gourhan, dans Le Geste et la parole (1964), a interprété l'émergence de l'espèce humaine comme l'extériorisation de certaines des fonctions du corps animal en un corps social composé de systèmes techniques et symboliques, et comme la rétroaction de ce corps social sur le corps animal. Cela revient à dire qu'il y a eu cosuscitation entre le milieu, anthropisé par la technique et humanisé par le symbole, et l'espèce humaine, hominisée par son milieu ; ou qu'anthropisation, humanisation et hominisation furent corrélatives. Avec l'idée que l'humain associe nécessairement un corps animal individuel et un corps social (ou plutôt médial, car il n'est pas seulement techno-symbolique, mais éco-techno-symbolique), cela corrobore en outre la définition watsujienne de la médiance (füdosei 風土性) comme " moment structurel de l'existence humaine " (ningen sonzai no kōzō keiki 人間存在の構造契機), c'est-à-dire en somme la co-suscitation des deux "moitiés " (medietates) qui font un être humain : l'individu, et son milieu.

La mésologie peut aujourd'hui faire la synthèse de ces diverses approches avec le concept de trajection (tsütaika 通態化 ${ }^{1}$ ), qui résume l'idée suivante : la réalité d'un milieu humain est produite par la manière dont, historiquement, une société humaine a saisi son environnement par les sens, par la pensée, par le langage et par l'action. Cette trajection est analogue à une prédication, dont le sujet $S$ est l'environnement, et le prédicat $\mathrm{P}$ le monde (au sens que l'on a vu plus haut) propre à la société en question. Les divers prédicats constitutifs de ce monde se ramènent à quatre grandes catégories $^{2}$ : ressources, contraintes, risques et agréments. La réalité d'un milieu

1. Pour plus de détails, voir Berque $(2000,2010)$ et également le site " mésologiques. com ». Il faut prendre garde que le sujet du logicien (S), i.e. ce dont il s'agit, c'est l'objet du physicien ; objet qui, dans le dualisme, s'oppose au sujet des sciences humaines.

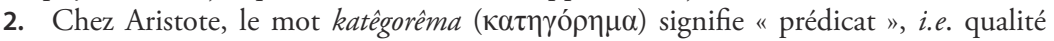
attribuée à un objet, donc attribut de cet objet. Cette catégorisation commençant avec la vie, elle est très largement inconsciente et prélangagière. Nous sommes par exemple inconscients que notre œil catégorise (perçoit) la longueur d'onde électromagnétique 
peut donc se résumer par la formule $r=S / P$, qui se lit : la réalité $r$, c'est l'environnement $S$ saisi en tant que monde P. C'est dans ce rapport que l'environnement $(S)$ est trajecté par l'histoire en un certain milieu $(S / P)$.

$\mathrm{Au}$ cours de cette trajection, la réalité $\mathrm{S} / \mathrm{P}$ ne cesse d'être réinterprétée par les générations nouvelles, au moyen de nouveaux prédicats. Elle devient $(\mathrm{S} / \mathrm{P}) / \mathrm{P}$ ', et ainsi de suite : $\left(((\mathrm{S} / \mathrm{P}) / \mathrm{P})^{\prime}\right) / \mathrm{P}$ ”)/P'”... Le milieu est ainsi de plus en plus mondanisé ; autrement dit, de moins en moins réductible à l'environnement naturel (le $S$ initial). Or l'existence humaine, instance énonciatrice des prédicats $\mathrm{P}$, est prise avec les choses dans cette trajection qui l'a co-suscitée elle-même, et ne peut s'en abstraire qu'au prix d'une mutilation.

Dans les sociétés traditionnelles, cependant, l'expérience historique tempère cette prédicativité, car le fondement naturel $(\mathrm{S})$ du monde humain $(\mathrm{P})$ rappelle toujours sa présence. L'expérience y nourrit une raison trajective toujours attentive à ce fondement naturel. Le monde moderne en revanche, imbu de ses techniques et de son rationalisme objectifiant, qui dégage le sujet humain de tout lien existentiel avec les choses muées en objets, tend à s'absolutiser dans sa propre mondanité, donc à perdre toute base dans la nature.

Cette mondanité asymptotique, c'est ce qui produit les catastrophes comme le San-ichi-ichi.

\section{Oubli de l'histoire et désastre humain}

Alors que tout scientifique eût pu vérifier que, sur la côte où fut implantée la centrale de Fukushima, des tsunamis de plus de sept mètres avaient été recensés au cours de l'histoire et même dans un passé récent, les concepteurs de la centrale choisirent de limiter la digue protectrice à sept mètres. Au mépris de l'histoire, l'implantation littorale elle-même résultait de la réduction du milieu à une seule catégorie prédicative : la ressource (l'eau de

$\lambda=700 \mathrm{~nm}(\mathrm{~S})$ en tant que rouge (P), tandis que l'œil de la vache ne perçoit pas le rouge. « $\lambda=700 \mathrm{~nm}$ » est affaire d'environnement, « rouge » est affaire de milieu. 
mer permettant de refroidir les réacteurs), forclosant ${ }^{3}$ les trois autres et en particulier le risque (de tsunami).

C'est pour la même raison que les banlieues de Sendai 仙台 se sont étendues dans la plaine exposée aux tsunamis. Dans le dualisme et l'économicisme propres à la mondanité moderne, la terre, perdant toute qualité historique, c'est-à-dire en cessant d'être un milieu, tend à n'être plus qu'une étendue physique réductible en mètres carrés de capital foncier. Paradigmatiquement en effet, dans la spatialité moderne, le capital règne sur une étendue neutre (l'extensio cartésienne) : cet " espace universel » que revendiqua le mouvement moderne en architecture. L'extensio est purement matérielle, et totalement quantifiable, comptable et comptabilisable en taux de profit par mètre carré. La rente étant supérieure en centre ville, cette dernière se décompose et éclate en urbain diffus, pour le plus grand profit de ceux qui réalisent la culbute entre le prix d'achat et le prix de vente des mêmes mètres carrés, ou abaissent le coût de la rente en allant construire des grandes surfaces commerciales toujours plus vastes toujours plus loin de la ville.

Étudiant ce phénomène du point de vue de ses effets sociaux, Miura Atsushi 三浦展, dans Fasuto-füdoka suru Nippon ファスト風土化する日本 (Notre füdo devient MacDo, 2004), a pu parler de fast-füdö̈sation. Autrement dit, le milieu devient non-lieu : il n'est plus que cette extensio toisée comme un objet par un sujet auto-institué dans l'abstrait, que, du point de vue ontologique, Heidegger a dénoncée dès Être et temps (Sein und Zeit, 1927), parlant à cet égard de "privation de monde " (Entweltlichung $)^{4}$.

Réduire par abstraction le milieu à la seule catégorie "ressource " (une source de profit par mètre carré pour le capital) au détriment des trois autres, qui sont concrètement non moins constitutives d'un monde humain, cela n'est évidemment pas sans effet sur le paysage. Celui-ci est littéralement tué, tout l'environnement devenant insipide (sappükei 殺風景) ${ }^{5}$. Mais ce que traduit cette " mort du paysage " (Dagonet, 1982) va bien au delà de

3. Forclore (foris claudere, lock out) : expulser hors de la conscience, et en fermer la porte.

4. Sur ce thème, en particulier sous le rapport du capital et du travail, on pourra lire Franck Fischbach (2011).

5. Ce mot, qui se prononce en chinois shäfềngjing, signifie littéralement " tue-paysage ". Il a été créé par le poète Li Shangyin 李商隠 (813-858). 
l'esthétique. Comme le montre l'étude de Miura citée plus haut, c'est bien de " tue-milieu», de sappūdo 殺風土 qu'il s'agit.

Qu'est-ce que le tue-milieu ? C'est l'oubli de l'histoire (qui donne sens au milieu) dans la décomposition de celui-ci (la chair de l'histoire) en de simples éléments physiques purement quantifiables, par arrêt sur objet au moment présent ${ }^{6}$. Après le San-ichi-ichi, la presse n'a pas manqué de rapporter que les villages où l'on n'avait pas négligé les enseignements de l'histoire, en ne bâtissant pas d'habitations au dessous de la limite atteinte par de précédents tsunamis, avaient été épargnés par celui du 11 mars. Cela, en dépit de l'attrait que représentait la proximité de la mer en tant que ressource. La raison trajective, enracinée dans le milieu et dans l'expérience historique de celui-ci, commandait d'équilibrer les quatre catégories qui fondent la mondanité humaine dans l'environnement naturel. Ailleurs, la privation de monde propre au rationalisme objectifiant de la modernité, plus particulièrement à celui du capitalisme, a au contraire commandé de faire le même choix qu'à Fukushima : privilégier la ressource en forclosant les trois autres catégories, donc en dépeçant le milieu pour n'en faire qu'une table rase définie par le seul profit.

Cette forclusion du milieu dans l'oubli de l'histoire ne joue pas qu'envers le passé, mais aussi envers l'avenir. Dans l'état de nos techniques, nous ne savons pas éliminer la radioactivité. Tous les retraitements dont nous sommes capables se bornent à la confiner dans des capsules au contenu hyperradioactif, que nous léguons à la postérité pour des centaines de milliers d'années (bien plus que l'âge de notre espèce). Cette forclusion de l'avenir au seul bénéfice du présent est, en termes temporels, exactement homologue à ce qu'est en termes spatiaux la forclusion de notre empreinte écologique. Ontologiquement, elle relève de cet " être vers la mort " (Sein zum Tode) purement individualiste que Watsuji dénonça chez Heidegger, en lui opposant l'" être vers la vie " (sei e no sonzai 生への存在) de la médiance humaine .

On aura compris que, du point de vue mésologique, ni l'urbain diffus ni le nucléaire ne sont défendables. Ils relèvent de la même abstraction

6. Sur ce thème, voir Berque (2000).

7. Sur ce thème, voir les actes du colloque «Être vers la vie » dans Ebisu (Berque et alii., 2009). 
mutilante, celle du sujet moderne hors de tout milieu et de toute histoire. C'est cette abstraction, plutôt que le tsunami puisqu'elle lui est antérieure, qui est la cause première de la catastrophe du San-ichi-ichi. Celui-ci n'aura fait que la révéler. C’est dire aussi que cette abstraction, libératrice au début, s'est retournée en un désastre humain plus profond que l'événement localisé du San-ichi-ichi; car ses effets continuent et s'amplifient à l'échelle de la planète. L'environnement terrestre connaîtra toujours des tsunamis, des tornades ou des avalanches; mais si l'humanité ne retrouve pas la raison trajective de son propre milieu, les catastrophes à venir seront toujours pires.

Palaiseau, 31 janvier 2012

\section{Bibliographie}

BERQUE Augustin, 2000

Écoumène. Introduction à l'étude

des milieux humains, Paris, Belin

[trad. japonaise : Fūdogaku josetsu

風土学序説, éd. Chikuma shobō

筑摩書房, 2002].

\section{BERQUE Augustin,}

BOUTRY-STADELMANN Britta,

FROGNEUX Nathalie,

UZUKI Sadami, 2009

"Être vers la vie» (Actes du colloque de

Cerisy), Ebisu 40-41 : 5-224.

\section{BERQUE Augustin, 2010}

Milieu et identité humaine. Notes

pour un dépassement de la modernité,

Paris, éd. Donner lieu.
DAGOGNET François (dir.), 1982

Mort du paysage?, Seyssel,

Champ Vallon.

FISCHBACH Franck, 2011

La privation de monde.

Temps, espace et capital, Paris, Vrin.

MIURA Atsushi 三浦展, 2004

Fasuto-füdoka suru Nippon ファスト風土 化する日本 (Notre fūdo devient MacDo), Tokyo, Yōsensha 洋泉社. 